\title{
Insegurança alimentar entre beneficiários de programas de transferência de renda ${ }^{1}$
}

\author{
Food insecurity among recipients \\ of government assistance
}

Francielle Richetti ANSCHAU2

Tiemi MATSUO3

Ana Maria SEGALL-CORRÊA ${ }^{4}$

RE S U M O

\section{Objetivo}

Analisar a proporção de insegurança alimentar domiciliar entre a população beneficiária de programas de transferência de renda e os fatores relacionados a essa condição.

\section{Métodos}

Estudo de delineamento transversal, cuja amostra foi constituída por 421 famílias beneficiárias, residentes no município de Toledo, Paraná. Os dados foram coletados entre setembro de 2006 e fevereiro de 2007, em entrevistas domiciliares, por meio da Escala Brasileira de Insegurança Alimentar e do Questionário de Classificação Econômica, ambos incluídos em questionário sociodemográfico. Para análise das variáveis, foram aplicados os testes de Qui-quadrado, razão de chances, regressão logística multivariada.

\section{Resultados}

A insegurança alimentar esteve presente em 74,6\% dos domicílios, 5,9\% na forma grave e 23,8\% moderada. Segundo o teste de Qui-quadrado, as variáveis independentes que mostraram associação com a insegurança foram renda per capita, classe econômica $D$ ou $E$, presença de menores de 18 anos, 7 ou mais membros no domicílio, baixa escolaridade e desemprego ou trabalho informal do chefe. Na regressão logística multivariada, considerando a variável dependente a condição de segurança alimentar/insegurança leve, mantiveram-se associadas à insegurança moderada/grave a classe econômica $D / E(O R=2,88)$, presença $5 / 6$ moradores $(O R=2,90)$ e 7 ou mais $(\mathrm{OR}=3,05)$, trabalho informal ou desemprego do responsável pelo domicílio $(\mathrm{OR}=1,87)$.

1 Artigo elaborado a partir da dissertação de FR ANSCHAU, intitulada "Insegurança alimentar de beneficiários de programas de transferência de renda". Universidade Estadual de Londrina; 2008

2 Universidade Paranaense, Instituto Superior de Ciências Biológicas, Médicas e da Saúde. Av. Parigot de Souza, 3636, Jd. Prada, 85900-000, Toledo, PR, Brasil. Correspondência para/Correspondence to: FR ANSCHAU. E-mails:<fran_anschau@hotmail.com>; <franciellea@unipar.br>.

3 Universidade Estadual de Londrina, Centro de Ciências Exatas, Departamento de Estatística. Londrina, PR, Brasil.

${ }^{4}$ Universidade Estadual de Campinas, Faculdade de Ciências Médicas, Departamento de Medicina Preventiva e Social. Campinas, SP, Brasil. 
178 | FR ANSCHAU et al.

\section{Conclusão}

O fato de a transferência de renda ainda deixar em situação de insegurança alimentar um contingente grande de beneficiários $(74,6 \%)$ é explicado pela extrema condição de vulnerabilidade social a que essa população está submetida. Os resultados deste estudo sugerem a necessidade de políticas públicas multissetoriais, com enfoque prioritário para geração de emprego e de renda.

Termos de indexação: Fome. Pobreza. Programas e política de nutrição e alimentação. Segurança alimentar e nutricional.

\section{A B S T R A C T}

\section{Objective}

This study analyzed the rate of food insecurity among recipients of government assistance and other factors associated with their conditions.

\section{Methods}

This cross-sectional study included 421 families from the municipality of Toledo, Paraná State, receiving government assistance. Data was collected from September/2006 to February/2007 during home interviews using the Brazilian Food Insecurity Scale, Economic Classification Questionnaires and other sociodemographic indicators. Variables were analyzed by the chi-square test, odds ratio and multivariate logistic regression.

\section{Results}

Most (74.6\%) of the households experienced moderate (23.8\%) to severe (5.9\%) food insecurity. According to the chi-square test, the independent variables associated with food insecurity were per capita income, socioeconomic classes D or E, family members under 18, 7 or more people living in the household, low education level and head of family unemployed or doing informal work. According to multivariate logistic regression and considering mild food insecurity a dependent variable, the variables that remained associated with moderate and severe food insecurity were socioeconomic classes D or E (OR=2.88), 5 to 6 people living in the household $(O R=2.90)$ or 7 or more $(O R=3.05)$, and head of family unemployed or doing informal work $(O R=1.87)$.

\section{Conclusion}

The extreme social vulnerability of recipients of government assistance explains the high rate (74.6\%) of food insecurity in this population. The results of this study suggest the need of job creation programs.

Indexing terms: Hunger. Poverty. Nutrition programs and policies. Food security.

\section{N T R O D U Ç Ã O}

Os programas de transferência direta de renda têm sido estratégia de muitos países para o enfrentamento da Insegurança Alimentar (IA) e da fome ${ }^{1}$. No Brasil, as primeiras iniciativas aconteceram na cidade de Campinas, no início dos anos 1990, com o programa municipal de Renda Mínima; em Brasília, surgiu o Bolsa Escola e, posteriormente, já em 2001, o Bolsa Alimentação, do Ministério da Saúde, além de outros auxílios monetários vinculados ao programa "Comunidade Solidária". Em 2003, com a implementação das políticas do "Fome Zero", houve progressiva integração dos vários programas dispersos, culminando, em 2004, com a instituição pelo Gover- no Federal do Bolsa Família (BF). Com diretrizes mais estruturadas e com aumento significativo da cobertura populacional nos anos subsequentes, esse programa atingiu, em 2010, mais de 12 milhões de famílias. O objetivo central do BF é a promoção da segurança alimentar, com articulação de ações visando à superação da fome e da pobreza no País².

A implementação do BF foi progressivamente acompanhada por investigações cujos objetivos eram avaliar o seu impacto sobre os padrões de consumo e, também, sobre a segurança alimentar da população beneficiária ${ }^{3}$. Estudo realizado em 2005, com a inclusão de mais de 15 mil domicílios do Brasil, mostrou impacto positivo das transferências de renda ao comparar o 
perfil de gastos de famílias beneficiárias do BF com o das não beneficiárias. Em termos gerais, as primeiras apresentavam gastos maiores na compra de alimentos e outros bens essenciais, comparativamente às não beneficiárias, na mesma faixa de renda 4 . Outro estudo que analisou a Pesquisa Nacional por Amostra de Domicílios (PNAD) de 2004 mostrou que a cada 10 reais transferidos pelos Programas de Transferência de Renda (PTR) aumentava-se em $8 \%$ a chance de segurança alimentar no domicilio ${ }^{5}$. Os mesmos autores afirmam que é relevante a contribuição das transferências de renda para a queda da desigualdade, diminuição da pobreza e melhoria das condições sociais da população.

Nas últimas décadas, em várias partes do mundo, observou-se um aumento significativo das investigações sobre Segurança Alimentar, que abordam tanto o diagnóstico de populações vulneráveis à fome, como seus determinantes e consequências para sua saúde e bem-estar. Vários estudos contribuíram para maior compreensão sobre as dimensões da segurança alimentar, o que tem permitido o uso de indicadores de segurança/insegurança alimentar adequados para o acompanhamento e a avaliação das intervenções, bem como para comparações da situação de acesso aos alimentos ao longo do tempo ${ }^{6}$. Dessa forma, os gestores das políticas públicas voltadas ao combate da insegurança alimentar e da fome passaram a ter instrumentos para avaliar periodicamente os programas de maneira segura e eficaz, adotando intervenções que melhorem essas condições ${ }^{7}$.

Recentemente, com o auxílio da Escala Brasileira de Insegurança Alimentar (EBIA), surgiram várias iniciativas de avaliação dos programas sociais de combate à insegurança alimentar. A EBIA permite identificar a prevalência de segurança/insegurança alimentar, por meio de perguntas centrais fechadas sobre a experiência nos últimos três meses de insuficiência alimentar em seus diversos níveis de intensidade, podendo variar de situações leves, como a preocupação de que a comida possa vir a faltar, até aquelas graves, em que um adulto ou criança do domicílio tem restrições de acesso aos alimentos, chegando mesmo a passar um dia todo sem comer ${ }^{8}$.

A insegurança alimentar atinge centenas de milhões de pessoas no mundo 9 . No Brasil, em 2004, 34\% dos domicílios foram classificados como tendo algum tipo ou grau de restrição alimentar, atingindo cerca de 72 milhões de pessoas. A prevalência de domićlios com insegurança alimentar aumentava para $66 \%$, considerando aqueles onde residia algum beneficiário de programas de transferência de renda ${ }^{10}$. Em outros países, como Bolívia, Burkina Faso e Filipinas, em 2004, a IA estava presente em 70\%, 73\% e 35\% das moradias, respectivamente ${ }^{11}$. Nos Estados Unidos, entre os 47500 domicílios amostrados no ano de 2005 , cerca de $11 \%$ dos seus moradores sofriam com a insegurança alimentar, aproximadamente 12,6 milhões de indivíduos, e, entre aqueles que participavam de programas de assistência alimentar, a prevalência de insegurança alimentar foi duas vezes superior ${ }^{12}$.

A vulnerabilidade à insegurança alimentar é observada, principalmente, nos domicílios de baixa renda ${ }^{13}$, que têm pouca disponibilidade ou pouco acesso aos alimentos, condição de utilização biológica inadequada, além de distribuição desigual entre os membros da família ${ }^{9}$. Entretanto, essa relação da IA com os baixos rendimentos familiares nem sempre é observada. Muitas vezes, domicílios com renda acima da linha de pobreza são classificados como condição de insegurança alimentar e vice-versa ${ }^{14}$, o que mostra que há necessidade de se utilizarem outros instrumentos e indicadores de medida da segurança e insegurança alimentar, e não apenas os rendimentos familiares para identificar os grupos mais vulneráveis a essas condições, e de auxiliar, dessa forma, a correção das desigualdades na distribuição dos benefícios. A maior frequência de insegurança alimentar entre beneficiários de PTR, comparativamente aos não beneficiários, do mesmo estrato social, indica a necessidade de melhor entendimento das circunstâncias que caracterizam a vida daquelas famílias. 
Este estudo teve como objetivo analisar a proporção de insegurança alimentar domiciliar entre a população beneficiária de programas de transferência de renda e os fatores relacionados a essa condição, em um município do sul do Brasil, buscando responder à necessidade de informações que possam auxiliar na elaboração ou na melhoria das políticas públicas locais de alimentação e nutrição.

\section{MÉ TOD O S}

O estudo transversal sobre segurança alimentar de famílias beneficiárias de programas de transferência de renda foi realizado no município de Toledo, oeste do Paraná (PR), no período de setembro de 2006 a fevereiro de 2007. A população estimada no município em 2006 era de 107033 habitantes, o Índice de Desenvolvimento Humano (IDH), de 0,827, e o índice de Gini, de $0,550^{15}$.

A população de estudo foi composta por todas as 4525 famílias cadastradas e que recebiam o benefício correspondente a algum programa de transferência de renda no município: Bolsa Família, Bolsa Escola, Bolsa Alimentação, Auxílio Gás e/ou do Programa de Erradicação do Trabalho Infantil (PETI). O número de beneficiários e os endereços das famílias foram fornecidos por órgãos municipais responsáveis pelo controle e pelo repasse dos benefícios. O tamanho da amostra teve como base de cálculo a frequência esperada de $50 \%$, por ser aquela que resulta em um maior tamanho de amostra, erro de 5\% na estimativa e nível de confiança de $95 \%$, totalizando 384 famílias. Acrescentou-se ao total o percentual de $30 \%$ de possíveis perdas, resultando em aproximadamente 500 domicílios. Utilizou-se a técnica de amostragem sistemática proposta por Silva ${ }^{16}$ na relação de endereços ordenada de acordo com o tipo de benefício e valor. Adotou-se a constante de amostragem de 9,05 (4525/500), e o início casual sorteado foi 2,29. As famílias foram selecionadas tanto na área urbana quanto na rural do município, sem visar proporcionalidade entre as duas.
A coleta dos dados foi realizada por meio de visitas domiciliares, com duração média de 35 minutos. Em cada domicílio foi entrevistado um membro da família com idade superior a 18 anos. No caso da residência estar fechada, procedia-se novamente à visita por até duas vezes, em dias e horários diferentes da primeira visita. Após a terceira visita, o domicílio era excluído e considerado como perda. Aos participantes entrevistados foi solicitada a assinatura do termo de consentimento livre e esclarecido.

O questionário utilizado na coleta de dados foi pré-testado e composto por questões relativas ao local do domicílio (rural/urbana, bairro) e às características da família (número total de moradores no domicílio, número de moradores com idade inferior a 6 anos e a 18 anos, identificação do chefe do domicílio, total de moradores segundo idade, sexo, escolaridade e ocupação de cada morador). As demais variáveis pesquisadas foram renda familiar mensal, valor do benefício recebido (somados para cálculo de renda per capita), participação em PTR. A classificação econômica das famílias foi estabelecida de acordo com o formulário da Associação Brasileira de Empresas de Pesquisa (ABEP), que estima o poder de compra das famílias urbanas por meio da soma dos pontos referentes aos bens de posse da família e grau de instrução do chefe ${ }^{17}$.

A variável dependente, insegurança alimentar em seus diferentes níveis (leve, moderada ou grave), e a presença de segurança alimentar foram avaliadas a partir de um escore formado pela somatória de respostas afirmativas aos 16 itens da $\mathrm{EBIA}^{10}$. A EBIA foi validada com 15 perguntas; posteriormente, uma delas, relativa ao "adulto ter diminuído a quantidade de alimentos na refeição ou ter deixado de fazer alguma refeição", foi desdobrada em duas (perguntas 9 e 11), resultando em uma escala com 16 itens apenas para fins de aplicação do questionário ${ }^{10}$.

Para a classificação da situação de segurança alimentar dos domicílios, foram estabelecidos pontos de corte no escore de respostas 
afirmativas, considerando a EBIA recomposta com 15 itens: segurança alimentar (somatório(S)=0), Insegurança Alimentar Leve (IAL) $\left(\mathrm{S}_{1}=1-5 \mathrm{em}\right.$ domicílios com membros menores de 18 anos e $\mathrm{S}_{2}=1-3$ em domicílios sem moradores menores de 18 anos), Insegurança Alimentar Moderada (IAM) $\left(\mathrm{S}_{1}=6-10 \mathrm{com}\right.$ menores de 18 e $\mathrm{S}_{2}=4-6 \mathrm{sem}$ menores de 18), e Insegurança Alimentar Grave (IAG) $\left(\mathrm{S}_{1}=11-15 \mathrm{com}\right.$ menores de 18 e $\mathrm{S}_{2}=7-8 \mathrm{sem}$ menores de 18) ${ }^{18}$.

A insegurança alimentar leve indica que há preocupação que os alimentos no domicílio venham a faltar antes de haver condições de se adquirir mais, com possibilidade de já estar sendo afetada a qualidade da dieta para a família. $\mathrm{Na}$ IAM, a qualidade da alimentação já está comprometida para toda a família, e os membros adultos da família já tiveram restrição quantitativa de alimentos. A IAG ocorre quando adultos e crianças já têm reduzido o seu acesso quantitativo aos alimentos e há situação de fome na família'12,18.

O questionário de classificação econômica da ABEP utilizado envolve posse de bens duráveis, uso de serviço doméstico e educação do chefe da família. Esse índice estabelece uma pontuação de 0 a 5, de acordo com cada item assinalado, podendo a família se enquadrar nas seguintes classes: A1 (30-34 pontos), A2 (25-29 pontos), B1 (21-24 pontos), B2 (17-20 pontos), C (11-16 pontos), $\mathrm{D}$ (6-10 pontos) e $\mathrm{E}$ (0-5 pontos).

A consistência interna das respostas aos itens da EBIA, análise que indica se as respostas estão correlacionadas entre si, foi medida por meio do coeficiente de alfa de Cronbach, considerando adequados valores superiores a 0,85. As associações entre as variáveis independentes e a variável dependente foram estabelecidas utilizando-se os testes de Qui-quadrado e de Kruskal-Wallis. As variáveis que apresentaram valor de $p$ menor do que 0,20 foram analisadas no modelo de regressão logística multivariado. Foram considerados significativos os testes que apresentaram níveis descritivos menores que $0,05(p<0,05)$. Os dados da pesquisa de campo foram duplamente digitados no Programa Epi Info, versão 3.4 para Windows. A análise estatística foi realizada no programa Statistical Analysis System for Windows versão 8.2 .

Esta pesquisa foi aprovada pelo Comitê de Ética em Pesquisa da Universidade Estadual de Londrina com emissão do parecer 215/06.

\section{RESULTADOS}

A Escala Brasileira de Insegurança Alimentar foi aplicada em 421 (84,2\%) domicílios: $409(97,1 \%)$ no perímetro urbano e $12(2,9 \%)$ na área rural. Registraram-se 79 perdas (15,8\%): por óbito (1), por recusa a assinar o termo de consentimento (10), domicílios não localizados (28), famílias não localizadas após três tentativas (9), recusa na abordagem inicial (8), mudança de endereço (22), moradora presa (1). As respostas aos itens da EBIA tiveram alta consistência, confirmada pelo valor de alfa de Cronbach $(0,8975)$, o que garante alta confiabilidade aos resultados do instrumento de coleta de dados sobre segurança alimentar.

No município de Toledo (PR), aproximadamente $75,0 \%$ das famílias beneficiárias de PTR sofrem com a insegurança alimentar em diferentes níveis: 44,9\% referiram IAL, 23,8\%, IAM e 5,9\%, IAG. Aproximadamente $35,0 \%$ dos domicílios tinham 4 moradores e em $94,3 \%$ havia um ou mais moradores com idade inferior a 18 anos. Somando o valor do benefício à renda mensal, $67,2 \%$ das famílias apresentavam renda per capita menor que $R \$ 150,00$, ou seja, menos de meio salário-mínimo na época da pesquisa (menos de $R \$ 190,00)$. A renda per capita média mensal foi de $R \$ 132,78$ (Desvio-Padrão - DP - $R \$ 97,60$ ), evidenciando alta variabilidade. Todas as famílias encontravam-se nas classes C, D ou E, e $64,6 \%$ delas faziam parte da classe D. O chefe do domicílio na maioria das famílias era do sexo masculino $(75,1 \%)$; o analfabetismo ou o ensino fundamental incompleto foram observados em 79,4\% dos chefes, e o trabalho informal ou o desemprego foram relatados por $50,1 \%$ dos chefes (Tabela 1). 
182 | FR ANSCHAU et al.

Tabela 1. Caracterização das famílias beneficiárias dos programas de transferência de renda quanto à insegurança alimentar, às variáveis relacionadas à família e ao chefe do domicílio. Toledo (PR), 2006-2007.

\begin{tabular}{|c|c|c|c|c|}
\hline \multirow{2}{*}{ Caracterização } & \multirow{2}{*}{$\mathrm{n}$} & \multirow{2}{*}{ Frequência (\%) } & \multicolumn{2}{|c|}{ I.C. $95 \%$} \\
\hline & & & Lim. Inf. (\%) & Lim. Sup. (\%) \\
\hline \multicolumn{5}{|l|}{ (In)Segurança alimentar } \\
\hline Segurança alimentar & 107 & 25,4 & 21,4 & 29,9 \\
\hline Insegurança alimentar leve & 189 & 44,9 & 40,1 & 49,8 \\
\hline Insegurança alimentar moderada & 100 & 23,8 & 19,8 & 28,2 \\
\hline Insegurança alimentar grave & 25 & 5,9 & 4,0 & 8,8 \\
\hline \multicolumn{5}{|l|}{ Constituição familiar } \\
\hline 3 moradores ou menos & 93 & 22,1 & 18,3 & 26,4 \\
\hline 4 moradores & 149 & 35,4 & 30,9 & 40,2 \\
\hline 5 a 6 moradores & 142 & 33,7 & 29,3 & 38,5 \\
\hline 7 moradores ou mais & 37 & 8,8 & 6,3 & 12,0 \\
\hline \multicolumn{5}{|c|}{ Renda per capita (86 ausências de resposta) } \\
\hline $\mathrm{R} \$ 75,00$ ou menos & 84 & 25,1 & 20,6 & 30,1 \\
\hline $\mathrm{R} \$ 75,01$ a $\mathrm{R} \$ 100,00$ & 65 & 19,4 & 15,4 & 24,1 \\
\hline $\mathrm{R} \$ 100,01$ a $\mathrm{R} \$ 150,00$ & 76 & 22,7 & 18,4 & 27,6 \\
\hline $\mathrm{R} \$ 150,01$ ou mais & 110 & 32,8 & 27,9 & 38,2 \\
\hline \multicolumn{5}{|l|}{ Classificação econômica } \\
\hline Classe C & 135 & 32,1 & 27,7 & 36,8 \\
\hline Classe D & 272 & 64,6 & 59,8 & 69,1 \\
\hline Classe $\mathrm{E}$ & 14 & 3,3 & 1,9 & 5,6 \\
\hline \multicolumn{5}{|l|}{ Sexo do chefe } \\
\hline Masculino & 316 & 75,1 & 70,6 & 79,1 \\
\hline Feminino & 105 & 24,9 & 20,9 & 29,4 \\
\hline \multicolumn{5}{|l|}{ Escolaridade do chefe } \\
\hline Analfabeto & 52 & 12,4 & 9,4 & 16,0 \\
\hline Fundamental incompleto & 282 & 67,0 & 62,2 & 71,4 \\
\hline Fundamental completo & 35 & 8,3 & 5,9 & 11,5 \\
\hline Ensino médio incompleto & 23 & 5,5 & 3,6 & 8,2 \\
\hline Ensino médio completo & 28 & 6,7 & 4,5 & 9,6 \\
\hline Superior incompleto & 1 & 0,2 & 0,0 & 1,5 \\
\hline \multicolumn{5}{|l|}{ Vínculo empregatício do chefe } \\
\hline Formal & 183 & 43,5 & 38,7 & 48,4 \\
\hline Informal & 147 & 34,9 & 30,4 & 39,7 \\
\hline Não trabalha & 64 & 15,2 & 12,0 & 19,1 \\
\hline Aposentado & 27 & 6,4 & 4,3 & 9,3 \\
\hline
\end{tabular}

IC: Intervalo de Confiança; Lim. Inf.: Limite Inferior; Lim. Sup.: Limite Superior.

A condição da presença de moradores menores de 18 anos mostrou-se relacionada à maior frequência de insegurança alimentar. Nos domicílios, somente com moradores adultos a condição de segurança alimentar teve proporção de $54,2 \%$, duas vezes superior às famílias com crianças e adolescentes. Outra variável que esteve associada foi o número de membros no domicílio, indicando que em domićlios com maior número de pessoas, maior a frequência de insegurança alimentar moderada ou grave $(p=0,0453)$. (Tabela 2).

A escolaridade do chefe era relativamente baixa e apenas um deles havia cursado algum período do curso superior. A distribuição de insegurança alimentar variou entre as outras três categorias de escolaridade, indicando que quanto maior a escolaridade mais distante o domicílio da 
Tabela 2. Insegurança alimentar segundo variáveis relacionadas à família e aos dados do chefe do domicílio, famílias beneficiárias de programas de transferência de renda, Toledo (PR), 2006-2007.

\begin{tabular}{|c|c|c|c|c|c|c|c|c|c|}
\hline \multirow{3}{*}{ Variável } & \multicolumn{6}{|c|}{ Nível de (in)segurança alimentar } & & & \multirow{3}{*}{ Valor de $p^{c}$} \\
\hline & \multicolumn{2}{|c|}{ SA } & \multicolumn{2}{|c|}{ IAL } & \multicolumn{2}{|c|}{ IAMG } & \multicolumn{2}{|c|}{ Total } & \\
\hline & $\mathrm{n}$ & $\%$ & $\mathrm{n}$ & $\%$ & $\mathrm{n}$ & $\%$ & $n$ & $\%$ & \\
\hline \multicolumn{10}{|l|}{ Moradores $<18$ anos } \\
\hline Sim & 94 & 23,7 & 185 & 46,6 & 118 & 29,7 & 397 & 100 & 0,0017 \\
\hline Não & 13 & 54,2 & 4 & 16,7 & 7 & 29,2 & 24 & 100 & \\
\hline \multicolumn{10}{|l|}{ Membros domicílio } \\
\hline 3 ou menos & 28 & 30,1 & 45 & 48,4 & 20 & 21,5 & 93 & 100 & 0,0453 \\
\hline 4 & 45 & 30,2 & 65 & 43,6 & 39 & 26,2 & 149 & 100 & \\
\hline 5 a 6 & 30 & 21,1 & 61 & 43,0 & 51 & 35,9 & 142 & 100 & \\
\hline 7 ou mais & 4 & 10,8 & 18 & 48,6 & 15 & 40,5 & 37 & 100 & \\
\hline \multicolumn{10}{|l|}{ Sexo } \\
\hline Masculino & 84 & 26,6 & 145 & 45,9 & 87 & 27,5 & 316 & 100 & 0,2291 \\
\hline Feminino & 23 & 21,9 & 44 & 41,9 & 38 & 36,2 & 105 & 100 & \\
\hline \multicolumn{10}{|l|}{ Idade } \\
\hline 29 ou menos & 9 & 16,7 & 30 & 55,6 & 15 & 27,8 & 54 & 100 & 0,2338 \\
\hline 30 a 49 anos & 68 & 28,2 & 103 & 42,7 & 70 & 29,0 & 241 & 100 & \\
\hline 50 anos ou mais & 25 & 25,3 & 39 & 39,4 & 35 & 35,4 & 99 & 100 & \\
\hline \multicolumn{10}{|l|}{ Escolaridade } \\
\hline $1^{\text {a }}$ & 43 & 22,4 & 85 & 44,3 & 64 & 33,3 & 192 & 100 & 0,0176 \\
\hline $2^{b}$ & 31 & 21,8 & 67 & 47,2 & 44 & 31,0 & 142 & 100 & \\
\hline $3^{c}$ & 33 & 37,9 & 37 & 42,5 & 17 & 19,5 & 87 & 100 & \\
\hline \multicolumn{10}{|l|}{ Trabalho } \\
\hline Formal & 62 & 33,9 & 80 & 43,7 & 41 & 22,4 & 183 & 100 & 0,0003 \\
\hline Informal & 27 & 18,4 & 68 & 46,3 & 52 & 35,4 & 147 & 100 & \\
\hline Não trabalha & 7 & 10,9 & 30 & 46,9 & 27 & 42,2 & 64 & 100 & \\
\hline Aposentado & 11 & 40,7 & 11 & 40,7 & 5 & 18,5 & 27 & 100 & \\
\hline \multicolumn{10}{|c|}{ Renda familiar per capita (reais) } \\
\hline $\mathrm{R} \$ 75,00$ ou menos & 10 & 11,9 & 37 & 44,0 & 37 & 44,0 & 84 & 100 & $<0,0001$ \\
\hline $\mathrm{R} \$ 75,01$ a 100,00 & 7 & 10,8 & 33 & 50,8 & 25 & 38,5 & 65 & 100 & \\
\hline $\mathrm{R} \$ 100,01$ a 150,00 & 10 & 13,2 & 38 & 50,0 & 28 & 36,8 & 76 & 100 & \\
\hline$R \$ 150,01$ ou mais & 42 & 38,2 & 50 & 45,5 & 18 & 16,4 & 110 & 100 & \\
\hline \multicolumn{10}{|c|}{ Classificação econômica } \\
\hline Classe C & 53 & 39,3 & 62 & 45,9 & 20 & 14,8 & 135 & 100 & $<0,0001$ \\
\hline Classe D ou E & 54 & 18,9 & 127 & 44,4 & 105 & 36,7 & 286 & 100 & \\
\hline
\end{tabular}

${ }^{a}$ Analfabeto/Primário incompleto; ${ }^{b}$ Primário completo/5a a $8^{a}$ séria incompleta; ${ }^{\mathrm{c}} 5^{\mathrm{a}}$ a $8^{\mathrm{a}}$ série completa/Segundo grau incompleto e completo/Superior incompleto; ${ }^{\mathbf{d}}$ Valor de $p$ utilizando o Teste de Qui-quadrado, nível de significância 5\%.

SA: Segurança Alimentar; IAL: Insegurança Alimentar Leve; IAMG: Insegurança Alimentar Moderada ou Grave.

insegurança alimentar, em qualquer de suas categorias: IAL, IAM ou IAG. A forma de trabalho do chefe do domicílio também esteve associada à insegurança alimentar domiciliar, sendo o nível moderado ou grave o mais prevalente entre os responsáveis com trabalho informal $(35,4 \%)$ e entre aqueles que não trabalhavam (42,2\%). Não foi observada associação entre insegurança alimentar e a faixa etária do chefe $(p=0,2338)$, bem como entre insegurança alimentar e o sexo $(p=0,2291)$, mas notou-se uma tendência ao aumento da IAMG com o aumento de sua idade e entre chefes do sexo feminino (Tabela 2). 
184 | FR ANSCHAU et al.

A IAM ou IAG apresentou-se associada aos menores estratos de renda per capita. À medida que se reduz essa condição, a chance da família vir a apresentar IAMG aumenta, como observado entre as famílias com rendimento per capita de até $R \$ 75,00$. A mesma situação pode ser visualizada nas classes econômicas D ou E (Tabela 2).

As estimativas resultantes da análise multivariada (Odds Ratio - OR ajustado) mostraram que a chance de IAMG nas classes econômicas D ou E foi 2,88 vezes superior (IC95\%: 1,66-5,00) à classe C. Nas famílias com 5 ou mais membros, a razão de chances para aquela condição foi cerca de 3 vezes maior em relação àquelas com até 3 moradores. Em domicílios onde o chefe trabalha de maneira informal ou está desempregado, as chances para IAMG aumentam em relação à família cujo chefe está inserido no mercado formal ou é aposentado $(O R=1,87)$. As demais variáveis analisadas (menores de 18 anos e sexo do chefe do domicílio) não apresentaram razão de chance elevada para a condição de insegurança alimentar moderada ou severa nos domicílios (Tabela 3).

\section{I S C U S S Ã O}

A insegurança alimentar é um fenômeno observado em todo o mundo, em países em desenvolvimento ou desenvolvidos, comprometendo a saúde e o bem-estar de 963 milhões de pessoas $^{19}$. Vários estudos, no Brasil e em outros países, têm mostrado alta prevalência de insegurança alimentar entre usuários de programas sociais. Entre refugiados africanos nos Estados Unidos e participantes de programas de assistência alimentar, 16\% das famílias apresentaram insegurança alimentar moderada ou grave ${ }^{20}$, frequência que pode ser considerada baixa, sugerindo a possibilidade de maior disponibilidade de alimentos referente ao seu país de origem ou,

Tabela 3. Razão de chances (OR) bruta e ajustada pela regressão logística multivariada para insegurança alimentar moderada ou grave (IAMG), Toledo (PR), 2006-2007.

\begin{tabular}{|c|c|c|c|c|c|c|c|c|c|c|}
\hline \multirow{2}{*}{ Variáveis $^{\text {a }}$} & \multicolumn{2}{|c|}{ IAMG } & \multirow{2}{*}{ Valor $p$} & \multirow{2}{*}{$\mathrm{OR}_{\text {bruto }}$} & \multirow{2}{*}{\multicolumn{2}{|c|}{ [IC 95\%] }} & \multirow{2}{*}{$\mathrm{OR}_{\text {ajustado }}$} & \multirow{2}{*}{\multicolumn{2}{|c|}{$[$ IC 95\% $]$}} & \multirow{2}{*}{ Valor $p$} \\
\hline & $n$ & $\%$ & & & & & & & & \\
\hline \multicolumn{11}{|l|}{ Classificação econômica } \\
\hline Classe C & 20 & 14,8 & $<0,001$ & 1,00 & & & 1,00 & & & \\
\hline Classe D ou E & 105 & 36,7 & & 3,34 & 1,96 & 5,68 & 2,88 & 1,66 & 5,00 & $<0,001$ \\
\hline \multicolumn{11}{|c|}{ Menores de 18 anos no domicílio } \\
\hline Sim & 118 & 29,7 & 0,954 & 1,03 & 0,42 & 2,54 & 0,62 & 0,21 & 1,82 & 0,382 \\
\hline Não & 7 & 29,2 & & 1,00 & & & 1,00 & & & \\
\hline \multicolumn{11}{|c|}{ Número de pessoas na família } \\
\hline Até 3 & 20 & 21,5 & 0,035 & 1,00 & & & 1,00 & & & \\
\hline 4 & 39 & 26,2 & & 1,29 & 0,70 & 2,39 & 1,89 & 0,91 & 3,91 & 0,086 \\
\hline 5 e 6 & 51 & 35,9 & & 2,05 & 1,12 & 3,73 & 2,90 & 1,40 & 6,01 & 0,004 \\
\hline 7 ou mais & 15 & 40,5 & & 2,49 & 1,09 & 5,66 & 3,05 & 1,21 & 7,69 & 0,018 \\
\hline \multicolumn{11}{|l|}{ Sexo do chefe } \\
\hline Masculino & 87 & 27,5 & 0,092 & 1,00 & & & 1,00 & & & \\
\hline Feminino & 38 & 36,2 & & 1,49 & 0,93 & 2,38 & 1,51 & 0,90 & 2,53 & 0,116 \\
\hline \multicolumn{11}{|l|}{ Trabalho do chefe } \\
\hline Formal ou aposentado & 46 & 21,9 & $<0,001$ & 1,00 & & & 1,00 & & & \\
\hline Informal ou não trabalha & 79 & 37,4 & & 2,13 & 1,39 & 3,28 & 1,87 & 1,18 & 2,95 & 0,007 \\
\hline
\end{tabular}

a Variável analisada no modelo de regressão logística porque apresentou valor de $p<0,20$ com (in)segurança alimentar avaliada em três categorias (IA, IAL e IAMG).

OR: Odds Ration; IAMG: Insegurança Alimentar Moderada ou Grave; IC: Intervalo de Confiança; IA: Insegurança Alimentar; IAL: Insegurança Alimentar Leve; IAMG: Insegurança Alimentar Moderada ou Grave. 
antes de serem incluídos no programa, indicando viés de resposta consequente da melhora no acesso aos alimentos. Já no Canadá, 70\% das mulheres usuárias de programas de assistência alimentar relataram problemas de acesso aos alimentos em termos de qualidade e/ou de quantidade, configurando, nesse último caso, situação de fome no domicílio ${ }^{21}$. Tanto a Pesquisa Nacional por amostra de Domicílios (PNAD) de $2004^{10}$ quanto a Pesquisa Nacional de Demografia e Saúde (PNDS) de $2006^{22}$ mostraram que os beneficiários de PTR tinham pior condição de acesso aos alimentos que os não beneficiários.

Segundo dados da PNAD $2004^{10}$, os níveis de insegurança alimentar - leve, moderado ou grave - acometeram $66 \%$ dos domićlios com pelo menos um morador beneficiário de PTR. A IAM $(26 \%)$ e IAG $(14,9 \%)$ foram superiores àquelas observadas entre beneficiários em Toledo também em população de beneficiários de transferência de renda. Resultados muito parecidos, embora de menor magnitude, foram encontrados na Pesquisa Nacional de Demografia e Saúde-2006²2. Em domicílios onde residiam mulheres em idade fértil e crianças menores de 5 anos, a IAMG entre beneficiários de programas de TR era de 32,5\%.

Pesquisa do Instituto Brasileiro de Análises Sociais e Econômicas (IBASE) de $2005^{23}$, que analisou amostra probabilística com 5 mil domicílios selecionados a partir do cadastro do programa Bolsa Família, mostrou que é de $83,1 \%$ a prevalência daqueles que estão passando por privações alimentares. A hipótese mais plausível para esses achados é a de que famílias beneficiárias de programas de tranferência de renda acumulam condições de maior vulnerabilidade, o que leva ao menor acesso a alimentos. Essa situação em geral é decorrente da marginalização ou exclusão social, com consequências graves para a saúde e bem-estar das pessoas. Se, de um lado, essas condições de vulnerabilidade levam a uma maior insegurança alimentar, de outro, se identificadas, podem melhor orientar a seleção dos beneficiários, o que acaba por constituir uma vantagem e um atestado de que os programas estão atingindo as populações de maior risco.
Entre os beneficiários de PTR em Toledo, prevaleceu a insegurança alimentar leve, que é caracterizada pela perda da qualidade da alimentação. Por outro lado, cerca de um terço dos domicílios estudados esteve exposto à IAMG (29,7\%), com privações e instabilidade de acesso aos alimentos, tanto do ponto de vista qualitativo como quantitativo. Essa situação em geral é decorrente da marginalização ou exclusão social, com consequências graves para a saúde e o bem-estar das pessoas. Deve constituir preocupação dos gestores locais das políticas públicas a existência de cerca de $6,0 \%$ de IAG entre os cadastrados nos PTR. A fome certamente está presente entre crianças desses domicílios, podendo afetar sua saúde, seu crescimento e seu desenvolvimento em longo prazo 24

Em Toledo, ao final das análises de regressão múltipla, observa-se que a chance de SA no domicílio aumenta em situação de melhor classificação econômica, menor número de pessoas no domicílio e presença de trabalho formal ou aposentadoria do chefe do domicílio. Famílias beneficiárias de transferência de renda e classificadas na classe econômica D ou E apresentaram chance de insegurança alimentar 3 vezes superior à classe C. Na PNDS-200622, os domicílios classificados no estrato econômico E tinham 213 vezes maior chance de insegurança alimentar grave comparativamente a segurança alimentar. Apesar de os dados não serem diretamente comparáveis, uma vez que a PNDS não se refere exclusivamente aos usuários dos programas sociais, eles mostram como em Toledo a pobreza influencia diretamente na segurança alimentar da população.

A chance de IAMG também se apresenta mais elevada em domicílios com 5 membros ou mais, corroborando resultados de outros estudos ${ }^{10,13,22,25}$. Essa relação é explicada pelo fato de que famílias grandes necessitam de mais recursos para compra de alimentos, e de que o crescimento familiar nem sempre é acompanhado de aumento da renda. Há referência, por outro lado, de que quando o impacto da composição familiar sobre a insegurança alimentar é controlado pelo efeito 
da renda, a primeira pode deixar de ser significativa $^{5}$, o que não ocorreu no presente estudo.

Outra condição significativamente associada a insegurança alimentar em Toledo foi a chefia do domicílio por mulheres, o que mostra que a segurança alimentar, do mesmo modo que outras condições sociais, apresenta uma relação de sexo. Observou-se que a chance de IAMG é $65 \%$ maior nesses domicílios, comparativamente aos chefiados por homens. Esse resultado também foi encontrado em análises dos dados da PNAD 2004, para a população geral do Brasil ${ }^{10}$.

Observou-se ainda neste estudo que, em domicílios onde o chefe está desempregado ou tem trabalho informal, a razão de chances para IAMG é maior ( $O R=1,87$, IC 1,18-2,95). As chances aumentadas para essa situação já foram observadas em estudo (OR=1,41, IC95\%: 1,07-1,87) com adolescentes ${ }^{25}$. O fato de o chefe ser aposentado ou ter trabalho formal resulta em maior acesso aos alimentos, reduzindo a preocupação familiar com a fome, uma vez que essas condições resultam em maior estabilidade econômica para a família.

Apesar dessa situação de maior insegurança alimentar das famílias beneficiárias dos programas de transferência de renda, não deve ser ignorado que o auxílio dos programas governamentais desempenha papel relevante na proteção social a esse grupo, especialmente ao aumentar ou desenvolver fontes alternativas de renda ${ }^{26}$. A tendência é a de que os PTR deixem as pessoas pobres um pouco menos pobres ${ }^{27}$, pois contribuem para melhoria das condições sociais dessa população, com redução da insegurança alimentar e da fome, especialmente entre os que vivem em situação de extrema pobreza 5 . Para essa população, têm sido relatados efeitos imediatos do PTR sobre suas condições de vida, entre eles a manutenção de ambientes familiares mais estruturados e saudáveis ${ }^{28}$.

Segundo Sampaio et al. ${ }^{29}$, para os participantes de grupos focais realizados por ocasião da validação da EBIA, o conceito de segurança alimentar está diretamente relacionado ao acesso, às condições de trabalho, saúde, educação, habitação e renda: condições que dignificam a condição humana e garantem o direito à alimentação adequada.

Na pesquisa linha de base Avaliação de Impacto do Programa Bolsa Família (AIBF), do Ministério do Desenvolvimento Social e Combate à Fome ${ }^{30}$, desenvolvida no ano de 2005 com 15 mil famílias beneficiárias e não beneficiárias, foi observado que a transferência de renda tem efeitos no bem-estar das famílias e crianças pobres, com impacto positivo na educação, vestuário e saúde infantil. Observou-se nessa investigação maior dispêndio de recursos na compra de alimentos ( $\mathrm{R} \$ 388,22 / a n o$ nas famílias em extrema pobreza e $R \$ 278,12$ /ano nas pobres), com consequente efeito positivo sobre a segurança alimentar de famílias beneficiárias de programas de transferência de renda.

Outras avaliações realizadas no país apontam a efetividade dos programas de transferência de renda ${ }^{27,30-32}$. Em Camaçari $(B A)^{31}$, observou-se que quando efetuado o pagamento do benefício, a família consegue administrar melhor suas necessidades básicas e, consequentemente, sua condição de vulnerabilidade. Resultados também indicam que os domicílios mais necessitados são os selecionados para receberem o benefício, o que contribuiu para a redução da pobreza ${ }^{27}$, aumento do poder de compra e melhoria das condições de segurança alimentar familiar.

Na realidade brasileira atual, considera-se que seja muito difícil uma família sair da extrema pobreza sem auxílio estatal. Por essa razão, o governo federal lançou programas sociais com propostas de erradicação da miséria e combate à pobreza, considerando ser alto o custo social dessas condições ${ }^{32}$.

O presente estudo apresentou algumas limitações. A primeira delas relativa ao seu delineamento transversal e ao fato de não se ter estudado famílias não beneficiárias, condições que dificultam as análises de associação causal. Também não foram consideradas neste estudo as diferenças de tempo de recebimento dos bene- 
fícios de transferência de renda. Outra limitação foi a desproporcionalidade entre beneficiários residentes em área rural e urbana do município, o que impediu a comparação das características de vulnerabilidade, possivelmente diferentes entre esses dois grupos.

Diante da relevância para o país dos programas sociais com foco nas populações mais vulneráveis, dentre eles os de transferência de renda, recomendam-se novos estudos que possam aprofundar a compreensão das condições de vida, saúde e nutrição dessas populações e a efetividade das políticas para mudança do cenário atual.

\section{O N CLUS Ã O}

Em Toledo, são altas as proporções de insegurança alimentar moderada e grave em domicílios onde residem beneficiários de algum PTR, refletindo o grau de marginalização social e vulnerabilidade à fome a que muitas dessas famílias estão submetidas. Os resultados obtidos indicam associação positiva entre os rendimentos familiares e a condição de insegurança alimentar, com maior chance para essa condição nos domicílios com renda per capita inferior a meio salário-mínimo. A classificação econômica D ou E, a presença de 5 ou mais membros no domicílio e o trabalho informal ou desemprego do chefe do domicílio também se apresentaram associados à condição de insegurança alimentar. Diante disso, as políticas de segurança alimentar e nutricional devem ter como objetivo final a redução da desigualdade, buscando resgatar os valores éticos, de equidade, cidadania, solidariedade e respeito à cultura. Isso não pode ser obtido apenas com a ação do Estado, são necessárias vontade e determinação de toda a sociedade.

Mesmo reconhecendo a importância das políticas de transferência de renda e outras de caráter emergencial, julga-se necessário o reforço das políticas públicas estruturantes, entre elas as políticas de promoção da igualdade de gênero, de geração de emprego, de acesso à terra e de recuperação do poder aquisitivo do salário-mínimo, que teriam impacto na redução da pobreza em geral, mas sobretudo nos estratos de extrema pobreza onde estão os beneficiários dos PTR. Pesquisas com beneficiários desses programas são ainda necessárias, sobretudo para avaliar e melhorar sua efetividade. Além disso, há necessidade de estudos sobre estratégias familiares para o enfretamento da insegurança alimentar e sobre o uso e modo de relacionamento das famílias com as ações das políticas públicas de combate à fome.

\section{A GRADECIMENTOS}

À Selma Maffei de Andrade, do Programa de Mestrado em Saúde Coletiva da Universidade Estadual de Londrina, pelas contribuições à redação.

\section{COLABORADORES}

FR ANSCHAU participou da concepção e desenho, análise e interpretação dos dados e da redação. T MATSUO e AM SEGALL-CORRÊA participaram da análise e interpretação dos dados e da redação.

\section{REFERÊ NCIAS}

1. Zimmermann C. Nota da relatoria nacional para o direito à alimentação adequada e terra rural sobre o programa bolsa família. Montes Claros: Plataforma Brasileira de Direitos Humanos, Econômicos, Sociais, Culturais e Ambientais; 2007 [acesso 2007 out 20]. Disponível em: <http://www. direitos. org.br>.

2. Brasil. Ministério do Desenvolvimento Social e Combate à Fome. Bolsa família. Brasília: MDS; 2010 [acesso 2007 set 15]. Disponível em: <http://www. mds.gov.br>.

3. Soares S, Sátyro N. O programa bolsa família: desenho institucional, impactos e possibilidades futuras. Brasília: Instituto de Pesquisa Econômica e Aplicada; 2009 [acesso 2011 out 15]. Disponível em: <http://www.ipea.gov.br>.

4. Paes-Souza R, Vaitsman J, organizadores. Metodologias e instrumento de pesquisas de programas do MDS. Brasília: Ministério do Desenvolvimento Social e Combate à Fome; 2007 [citado 2008 jun 22]. Disponível em: <http://bvsms.saude.gov.br>. 
188 | FR ANSCHAU et al.

5. Segall-Corrêa AM, Marin-León L, Helito H, Perez-Escamilla R, Santos LMP, Paes-Sousa R. Transferência de renda e segurança alimentar no Brasil: análise dos dados nacionais. Rev Nutr. 2008; 21(Supl 1):39-51.

6. Pereira RA, Santos LMP. A dimensão da insegurança alimentar. Rev Nutr. 2008; 21(supl 1):7-13.

7. Melgar-Quiñonez $H$, Hackett M. A Medida da segurança alimentar: a experiência mundial. Rev Nutr. 2008; 21(Supl 1):27-37. doi: 10.1590/S14 15-52732008000700004.

8. Segall-Corrêa AM, Perez-Escamilla R, Marin-Leon L, Yuyama L, Vianna RPT, Coitinho D, et al. Evaluation of household food insecurity in Brazil: validity assessment in diverse sociocultural settings. Concurso RedSan 2007. Brasília: MDS; 2009. [cited 2009 Aug 12]. Available from: <http://www.rlc. fao.org>.

9. Webb P, Coates J, Frongillo EA, Rogers BL, Swindale A, Bilinsky P. Measuring household food insecurity: why it's so important and yet so difficult do. J Nutr. 2006; 136(Suppl 1):1404-8.

10. Instituto Brasileiro de Geografia e Estatística. Pesquisa Nacional por Amostra de Domicílios. Aspectos complementares de educação e acesso a transferências de renda de Programas sociais. Rio de Janeiro: IBGE; 2006. [acesso 2007 set 20]. Disponível em: <http://www.ibge.gov.br>.

11. Melgar-Quiñonez HR, Zubieta AC, MkNelly B, Nteziyaremye A, Gerardo MFD, Dunford C. Household food insecurity and food expenditure in Bolivia, Burkina Faso and the Philippines. J Nutr. 2006; 136(Suppl 1):1431-7.

12. Nord M, Andrews M, Carlson S. Measuring food security in the United States. Household food security in the United States, 2005. Washington (DC): USDA; 2006. [cited 2007 Dec 15]. Disponível em: <http://www.ers.usda.gov>.

13. Salles-Costa R, Pereira RA, Vasconcellos MTL, Veiga GV, Marins VMR, Jardim BC, et al. Associação entre fatores socioeconômicos e insegurança alimentar: estudo de base populacional na Região Metropolitana do Rio de Janeiro, Brasil. Rev Nutr. 2008; 21(Supl 1):99-109.

14. Hall B. Understanding food security data and methodology [Internet]. Walthan: Food Security Institute. Center on Hunger and Poverty; 2004. [cited 2007 May 26]. Available from:<http://www. centeronhunger.org>.

15. Instituto Brasileiro de Geografia e Estatística. Perfil dos municípios brasileiros. Rio de Janeiro: IBGE; 2007 [acesso 2007 nov 11]. Disponível em: <http: www.ibge.gov.br>.

16. Silva NN. Amostragem probabilística. $2^{a}$ ed. São Paulo: EDUSP; 2001.
17. Associação Brasileira de Empresas e Pesquisa. Critério de classificação econômica Brasil. São Paulo: ABEP; 2000 [acesso 2007 jun 20]. Disponível em: <http://www.abep.org/codigosguias/ABEP_ CCEB.pdf $>$.

18. Segall-Corrêa AM, Panigassi G, Sampaio MFA, Marin-León L, Perez-Escamilla R. Validación de instrumento de medida de la inseguridad alimentaria y hambre, en el contexto de las políticas brasileñas de combate el hambre. Persp Nutr Hum [Internet]. 2007 [acceso 2009 nov 30]; 2:89-102. Disponible en: <http://revinut.udea.edu.co/ separata/pdf/separata1.pdf>.

19. Food and Agriculture Organization of the United Nations. Food insecurity in the world. Economic crises: impacts and lessons learned. Rome: FAO, 2009 [cited 2010 Jul 15]. Available from: <http:// www.fao.org $>$.

20. Hadley C, Zodhiates A, Sellen DW. Acculturation, economics and food insecurity among refugees resettled in the USA: a case study of West African refugees. Public Health Nutr. 2006; 10(4):405-12.

21. Tarasuk VS. Household food insecurity with hunger is associated with women's food intakes, health and household circumstances. J Nutr. 2001; 131(10):2670-6.

22. Segall-Corrêa A M, Marín-León L, Panigassi G, Pérez-Escamilla R. Capítulo 12: Segurança alimentar no domicílio. In: Berquó E, Garcia S, Lago T, editores. Pesquisa Nacional de Demografia e Saúde da Criança e da Mulher - PNDS 2006: dimensões do processo reprodutivo e da saúde da criança. Brasília: Ministério da Saúde; 2009 [acesso 2010 ago 2]. Disponível em: <http://fef.unicamp.br>.

23. Salles-Costa R, Segall-Correa AM. Novas possibilidades de alimentação a caminho? Democracia Viva [Internet]. 2008 [acesso 2010 mar 9]; 39:68-73. Disponível em: <http://www.ibase.br>.

24. Cook JT, Frank DA, Levenson SM, Neault NB, Heeren TC, Black MM, et al. Child food insecurity increased risks posed by household food insecurity to young children's health. J Nutr. 2006; 136(4): 1073-6.

25. Gulliford MC, Mahabir D, Nunes C, Rocke B. Sefladminstration of a food security scale by adolescents: item functioning, socio-economic position and food intakes. Public Health Nutr. 2005; 8(7):853-60.

26. Rose DD. Interventions to reduce household food insecurity: a synthesis of current concepts and approaches for Latin America. Rev Nutr. 2008; 21(Suppl 1):159-73. doi: 10.1590/S1415-527320 08000700014.

27. Tavares PA, Pazello ET, Ferandes R, Camelo RS. Uma avaliação do programa bolsa família: focalização e 
impacto na distribuição de renda e pobreza. Brasília: Instituto de Pesquisa Econômica e Aplicada; 2009 [acesso 2011 out 16]. Disponível em: <http:// www.ipea.org.br>.

28. Sousa PA. Um macro na consolidação do estado de bem-estar brasileiro. Democracia Viva [Internet]. 2008 [acesso 2009 jun 25]; 39:18-23. Disponível em: <http://www.ibase.br>.

29. Sampaio MFA, Kepple AW, Segall-Corrêa AM, Oliveira JTA, Panigassi G, Kurdian Maranha L, et al. (In) segurança alimentar: experiência de grupos focais com populações rurais do Estado de São Paulo. Segurança Alimentar e Nutricional [Internet]. 2006 [acesso 2007 mar 22]; 13:64-77. Disponível em: <http://www.unicamp.br>.

30. Brasil. Ministério do Desenvolvimento Social e Combate à Fome. Avaliação do impacto do progra- ma bolsa família. Brasília: MDS; 2007 [acesso 2011 out 16]. Disponível em: <http://www.mds.gov.br>.

31. Dorneles DF. Percurso metodológico de análise do Programa Bolsa Família na RMS/Bahia: estudo de caso de Camaçari, Candeias e Simões Filho. Textos e Contextos [Internet]. 2010 [acesso 2012 abr 11]; 9(1):132-49. Disponível em: <http://www.revistas eletronicas.pucrs.br>.

32. Faria CT. Eficácia, eficiência e efetividade do Programa Bolsa Família no município de Cruz do Espírito Santo/PB. Rev Eletrônica Ciênc Sociais [Internet]. 2010 [acesso 2011 out 16]; 15:46-65. Disponível em: <http://www.cchla.ufpb.br>.

Recebido em: 28/10/2010

Versão final em: 14/2/2012

Aprovado em: 23/3/2012 
